\title{
Classificação Internacional para a Prática de Enfermagem: instrumental tecnológico para a prática profissional
}

\author{
International Classification for Nursing Practice: technological instrument for professional practice
}

Clasificación Internacional para la Práctica de Enfermería: intrumentral tecnológico para la práctica profesional

\author{
Maria Miriam Lima da Nóbrega', Telma Ribeiro Garcia' \\ 'Universidade Federal da Paraíba. Centro de Ciências da Saúde, Programa de Pós-Graduação em Enfermagem. João Pessoa, PB
}

Submissão: $05 / 05 / 2009$

Aprovação: 3 l/08/2009

\section{RESUMO}

Considera-se o uso de tecnologias de informação como instrumentos Que podem fazer com Que a prática de enfermagem se torne visível no conjunto de dados de saúde, locais, nacionais e internacionais, de modo a influenciar na elaboração de políticas de saúde e de educação. Aborda-se o desenvolvimento dos sistemas de classificação em enfermagem, com ênfase na Classificação Internacional para a Prática de Enfermagem - CIPE ${ }^{\circledR}$, como instrumental tecnológico para a prática profissional, Que tem como um dos principais critérios para sua construção o de ser suficientemente ampla e sensível à diversidade cultural, de modo que sirva aos múltiplos propósitos requeridos pelos distintos países onde será utilizada.

Descritores: Enfermagem; Sistema de classificação; Tecnologia da informação.

\section{ABSTRACT}

The use of information technologies are considered as instruments that can do that nursing practice becomes visible in health data, local, national and internationally, in way to influence the elaboration of health and education policies. The development of nursing classification systems is approached, with emphasis in the International Classification for Nursing Practice - ICNP ${ }^{\circledR}$, with technological instrument for professional practice, which has as one of the principal criteria for its construction to be sufficiently wide and sensitive to cultural diversity, so that it serves to the multiples purposes reQuested by the different countries where it will be used.

Descriptors: Nursing; Classification systems; Information technology.

\section{RESUMEN}

El uso de tecnologías de información es considerado como instrumentos que pueden hacer la práctica de enfermería visible en los datos de salud, local, nacional e internacionalmente, de manera a influir en la elaboración de políticas de salud y educación. Aborda-se el desarrollo de los sistemas de la clasificación en enfermería se aproxima, con énfasis en la Clasificación Internacional para la Práctica de Enfermería - CIPE ${ }^{\circledR}$, cono instrumental tecnológico para la practica profesional, Que tiene como uno de los criterios para su construcción el de ser suficientemente ancho y sensible a la diversidad cultural, para Que sirva a los múltiplos propósitos requeridos por los diferentes países dónde se la usará.

Descriptores: Enfermería; Sistemas de clasificación; Tecnología de información. 


\section{INTRODUÇÃO}

Segundo o Conselho Internacional de Enfermagem (CIE), neste início do século XXI, a saúde no mundo evolui em meio a uma grande agitação política e econômica. Os traumatismos, as enfermidades, as doenças endêmicas, as doenças crônico-degenerativas e os efeitos da pobreza, da fome, da guerra, entre outros, ameaçam a saúde e o bem-estar da população. Por outro lado, cada vez mais se dispõe de tecnologias de informação para processar e gerenciar dados Que melhorem a prática profissional, a administração, a formação, a investigação e a divulgação dos conhecimentos, entre os Quais os conhecimentos de enfermagem. Há muito tempo, tem-se considerado Que a articulação clara do exercício da Enfermagem é essencial para Que se reconheça plenamente a amplitude e a profundidade da profissão(l).

O uso de tecnologias de informação, entendidas como recursos não humanos (softwares ou hardwares), dedicadas ao armazenamento, processamento e comunicação da informação ${ }^{(2)}$, pode fazer com Que a prática de enfermagem se torne visível no conjunto de dados, locais, nacionais e internacionais, sobre a saúde, de modo a influenciar na elaboração de políticas, tais como as de saúde e de educação. As tecnologias de informação são, também, essenciais para a tomada de decisões eficazes e para uma prática de Qualidade, de forma Que os conhecimentos profissionais adQuiridos possam ajudar a conhecer e a compreender melhor os assuntos relacionados com a atenção à saúde.

A Enfermagem, nas últimas décadas, vem se preocupando com a utilização de um sistema de linguagem unificada de enfermagem Que permita acessar as informações da área nos sistemas de saúde. Por esta razão, os pesQuisadores da área têm chegado à conclusão Que a aplicação de normas de terminologia é essencial para o desenvolvimento de sistemas de informação Que facilitem a tomada de decisões clínicas e a prática baseada em evidências em enfermagem.

O CIE entende a necessidade de dados de enfermagem e reconhece Que é essencial contar com normas para representar a prática profissional nos sistemas de informação de saúde e para desenvolver uma compreensão, baseada em conhecimento, do trabalho da Enfermagem no contexto da atenção global à saúde ${ }^{(1)}$.

Um importante programa do CIE, destinado a integrar, no âmbito mundial, a infra-estrutura da informação sobre a política e a prática de atenção à saúde, é a Classificação Internacional para a Prática de Enfermagem $\left(\mathrm{CIPE}^{\circledR}\right)$. Este programa tem como meta adaptar o trabalho de desenvolvimento de uma terminologia de enfermagem às normas internacionais, e de maneira compatível com outras disciplinas da área da saúde ${ }^{(3)}$. Assim pensada, a CIPE ${ }^{\circledR}$ representa uma ferramenta ou instrumental tecnológico Que: facilita a comunicação das enfermeiras sobre sua prática, seja entre si, com outros profissionais e/ou com formuladores de políticas de saúde; facilita a padronização da documentação do cuidado prestado ao paciente; facilita o intercâmbio de dados entre populações, ambientes de prestação de cuidados, linguagens e lugares geográficos distintos; permite o uso desses dados para o planejamento e gerenciamento do cuidado de enfermagem, para a previsão de financiamentos e para a análise de resultados alcançados com a ação/intervenção de enfermagem, entre outros $^{(3)}$

Dessa forma, foi estabelecido como objetivo para este artigo o de discorrer sobre o desenvolvimento histórico dos sistemas de classificação em enfermagem, com ênfase na CIPE ${ }^{\circledR}$ como instrumental tecnológico para a prática profissional.

\section{SISTEMAS DE CLASSIFICAÇÃO EM ENFERMAGEM}

Como ocorre em Qualquer outra área, a Enfermagem, em seu ambiente de trabalho, utiliza um vocabulário técnico particular, a Que se pode denominar de linguagem especial, Que constitui uma modalidade de expressão do grupo e incorpora os conceitos, abstratos ou concretos, utilizados em sua atividade profissional. Esses conceitos se deixam reunir em grupos estruturados de tal modo que cada um fica ali definido pelo lugar Que ocupa respectivamente à posição dos demais, formando redes de termos inter-relacionados ${ }^{(4)}$.

No final da década de 80 do século XX, a Enfermagem já contava com certo número de sistemas de classificação dos termos da linguagem profissional, cujo desenvolvimento estava relacionado com alguma das fases do Processo de Enfermagem - a Taxonomia de Diagnósticos de Enfermagem da NANDA Internacional, a CIPE ${ }^{\circledR}$, o Sistema Comunitário de Saúde de Omaha, a Classificação de Intervenções de Enfermagem (NIC), a Classificação de Resultados de Enfermagem (NOC), entre outros ${ }^{(4)}$.

A despeito dos avanços alcançados, observava-se, ainda, a necessidade de uma terminologia partilhada no âmbito mundial para expressar os elementos da prática de enfermagem: o Que as enfermeiras fazem (ações/intervenções de enfermagem), face a determinadas necessidades humanas da clientela (diagnósticos de enfermagem), para produzir os resultados esperados (resultados de enfermagem). A utilização dessa terminologia deveria permitir, não somente descrever a prática profissional da Enfermagem, como, também, compará-la entre cenários clínicos, populações de clientes, áreas geográficas ou tempo distintos ${ }^{(5)}$.

Assim, por ocasião do $19^{\circ}$ Congresso Quadrienal do CIE, realizado em 1989, em Seul - Coréia, aprovou-se a resolução de estabelecer uma classificação para a prática de enfermagem, mobilizando as associações nacionais a ele filiadas. Inicialmente, foram propostos como objetivos para esta classificação os de fornecer uma ferramenta para descrever e documentar as práticas de enfermagem; usar essa ferramenta como base para a tomada de decisão clínica; e prover a profissão com um vocabulário e um sistema de classificação Que possam ser usados para incluir dados de enfermagem nos sistemas de informação computadorizados ${ }^{(5)}$.

Em 1996, o CIE publicou a Classificação Internacional para a Prática de Enfermagem - Versão Alfa: um marco unificador, contendo a Classificação de Fenômenos de Enfermagem e a Classificação de Intervenções de Enfermagem ${ }^{(6)}$. Essa versão foi traduzida para diversas línguas, inclusive para o português do Brasil. Na evolução, divulgouse a Versão Beta, em 1999, durante as comemorações dos cem anos do CIE. Em 200 I, o CIE publicou a Versão Beta 2 e, em 2005, a CIPE $^{\circledR}$ Versão 1.0.

A CIPE ${ }^{\circledR}$ está sendo desenvolvida como um marco unificador dos diversos sistemas de classificação em enfermagem, permitindo a configuração cruzada de termos de classificações já existentes e de outras Que forem desenvolvidas. Um dos principais critérios dessa classificação é o de poder ser suficientemente ampla e sensível à diversidade cultural, de modo Que sirva aos múltiplos propósitos requeridos pelos distintos países onde será utilizada ${ }^{(7)}$.

A utilização da CIPE $^{\circledR}$ Versão Beta 2 evidenciou Que a estrutura 
dessa classificação dificultava o alcance da meta de um sistema de linguagem unificada de enfermagem e não estava satisfazendo as necessidades das enfermeiras. Partindo dessa constatação, o Grupo de Aconselhamento Estratégico da CIPE $^{\circledR}$ desenvolveu uma investigação entre líderes mundiais no domínio de vocabulários usados em cuidados de saúde, com a finalidade de assegurar Que a próxima versão da CIPE ${ }^{\circledR}$, a Versão 1.0, fosse consistente com os vocabulários e normas existentes ${ }^{(5)}$.

\section{CIPE $^{\circledR}$ VERSÃO $\quad 1.0$}

A CIPE ${ }^{\circledR}$ Versão 1.0 tem múltiplos propósitos: identificar similaridades e diferenças entre diferentes representações, de modo a comparar dados de diferentes fontes; facilitar o desenvolvimento de vocabulários locais; preencher uma necessidade prática de construir sistemas de registro eletrônicos do paciente, com todos os benefícios de fazer parte de um sistema de linguagem unificada ${ }^{(8)}$.

A principal inovação foi Que, enQuanto a Versão Beta 2 estava estruturada em duas classificações (Fenômenos e Ações de Enfermagem), com um total de dezesseis eixos, a CIPEÂ Versão 1.0 contém uma única estrutura de classificação, organizada em sete eixos, definidos como: foco - área de atenção relevante para a Enfermagem; julgamento - opinião clínica ou determinação relacionada ao foco da prática de enfermagem; meios - maneira ou método de executar uma intervenção; ação - processo intencional aplicado a, ou desempenhado por um cliente; tempo - o ponto, período, momento, intervalo ou duração de uma ocorrência; localização - orientação anatômica ou espacial de um diagnóstico ou intervenção; e cliente - sujeito a Quem o diagnóstico se refere e Que é o beneficiário de uma intervenção de enfermagem ${ }^{(5)}$.

As vantagens dessa nova estrutura é Que a mesma simplifica extremamente a representação e resolve os problemas de redundâncias e ambiguidades de termos que eram inerentes à CIPE ${ }^{\circledR}$ Versão Beta $2^{(9)}$. O Modelo de Sete Eixos é destinado a facilitar a composição de afirmativas organizadas em grupos significativos, de modo Que se tenha acesso rápido a conjuntos de enunciados preestabelecidos de diagnósticos, intervenções e resultados de enfermagem - Catálogos CIPE ${ }^{\circledR}$. Os principais propósitos da elaboração desses Catálogos são os de construir sistemas de registro dos elementos da prática usando a CIPE ${ }^{\circledR}$, com todos os benefícios de fazer parte de um sistema de linguagem unificada; e tornar essa classificação um instrumental tecnológico que pode ser integrado à prática de enfermagem no local do cuidado ${ }^{(8,10-12)}$

Os Catálogos CIPE ${ }^{\circledR}$ podem estar direcionados tanto a clientelas específicas (indivíduo, família e comunidade), Quanto a prioridades de saúde específicas, relacionadas a condições de saúde, ambientes ou especialidades de cuidado e fenômenos de enfermagem.

Os Catálogos CIPE ${ }^{\circledR}$ podem originar conjuntos de dados a serem usados para apoiar e melhorar a prática clínica, o processo de tomada de decisão, a pesquisa e a formação profissional. Além disso, contribuirá para a expansão do uso da $\mathrm{CIPE}^{\circledR}$ no âmbito mundial, uma vez Que permite focalizar as variações culturais e linguísticas. Entretanto, esses Catálogos não substituem o julgamento clínico da enfermeira, Que será sempre essencial para dispensar cuidados individualizados aos pacientes e suas famílias ${ }^{(1)}$.

\section{CONSIDERAÇÕES FINAIS}

Sem sombra de dúvida, o esforço despendido na elaboração de sistemas de classificação dos termos da linguagem profissional tem contribuído para promover a autonomia da enfermeira no julgamento sobre as necessidades humanas da clientela, para facilitar o uso de conhecimentos específicos e para a realização de estudos sobre a Qualidade do cuidado de enfermagem.

Desde o lançamento da CIPE ${ }^{\circledR}$, várias pesQuisas e experiências de sua implementação na prática profissional estão em andamento no âmbito mundial. A CIPE ${ }^{\circledR}$ é uma terminologia dinâmica e, dessa forma, se beneficia com a participação contínua, seja local, nacional ou internacional. Acredita-se Que a documentação do cuidado de enfermagem utilizando a CIPE ${ }^{\circledR}$ proverá dados sistemáticos e recuperáveis sobre o cuidado à saúde, possibilitando maior visibilidade e reconhecimento social da profissão ${ }^{(10)}$.

Envolvidas com sua utilização e desenvolvimento, desde a implementação do Projeto CIPESC no Brasil, encaminhamos em 2007 uma proposta de criação de um Centro de Pesquisa e Desenvolvimento da CIPE ${ }^{\circledR}$ para ser acreditado pelo CIE. Em julho de 2007 o CIE aprovou o Centro de Pesquisa e Desenvolvimento da CIPE $^{\circledR}$ do Programa de Pós-Graduação em Enfermagem da Universidade Federal da Paraíba - Brasil (Centre for ICNP ${ }^{\circledR}$ Research and Development of the Federal University of Paraiba, Post-Graduate Program in Nursing - Brazil - An ICN Accredited Centre). Este Centro tem como missão apoiar o desenvolvimento contínuo da $\mathrm{CIPE}^{\circledR}$; promover o seu uso na prática clínica, na educação e na pesQuisa em enfermagem; e colaborar com o CIE e outros Centros semelhantes na transformação da CIPE ${ }^{\circledR}$ em uma terminologia de referência a ser usada mundialmente como instrumental tecnológico para fortalecer e ampliar os propósitos da profissão na assistência, na educação e na peseuisa.

\section{REFERÊNCIAS}

1. Conselho Internacional de Enfermeiros. Directrices para la preparación de Catálogos de la ICNP ${ }^{\circledR}$. Ginebra: ICN; 2008.

2. Alecrim E. O Que é Tecnologia da informação. Coluna Infowest; 2004. [Citado em 9 abr 2008]. Disponível em: http:// www.infowester.com/col I 50804.php

3. Conselho Internacional de Enfermeiros. Classificação Internacional para a Prática de Enfermagem Versão 1.0. São Paulo: Algol Editora; 2007.

4. Garcia TR, Nóbrega MML. Processo de enfermagem e os Sistemas de Classificação dos Elementos da Prática Profissional:

instrumentos metodológicos e tecnológicos do cuidar. In: Santos I, Figueiredo NMA, Padilla MICS, Cupillo AJ, Souza SROS, Machado WCA, organizadores. Enfermagem Assistencial no Ambiente Hospitalar: realidade, Questões, soluções - Série Atualização em Enfermagem. São Paulo: Atheneu; 2004. p. 37-63.

5. Conselho Internacional de Enfermeiros. Classificação Internacional para a Prática de Enfermagem Versão 1.0. Lisboa: Ordem dos Enfermeiros de Portugal; 2005.

6. International Council of Nurses. The International Classification for Nursing Practice: a unifying framework - the Alpha version. 
Geneva (Switzerland): ICN, 1996.

7. International Council of Nurses. ICNP ${ }^{\hat{a}}$ - International Classification for Nursing Practice - Beta. Geneva (Switzerland): ICN, 1999.

8. Coenen A. International Classification for Nursing Practice $\left(\mathrm{ICNP}^{\circledR}\right)$. Presented in: ICNP ${ }^{\circledR}$ Consortium Meeting, held during the ICN Conference. 30 May-Jun 2007. Yokohama, Japan. [Cited 4 jul 2007]. Available from: http://www.icn.ch/icnp-pres2007/ Introduction-ICNP-Consortium.html

9. International Council of Nurses. International Classification for Nursing Practice - ICNP ${ }^{\circledR}$ Version 1.0. Geneva: ICN; 2005.
10. International Council of Nurses. Nurse experts needed for ICNP ${ }^{\circledR}$ catalogue review. ICNP ${ }^{\circledR}$ Bulletin 2007; ( I).

11. Bartz C, Coenen A, Hardiker N, Jansen K. ICNP ${ }^{\circledR}$ catalogues. Presented in: ACENDIO Conference. 2007 apr 19-2 I. Amsterdam, Netherlands. [Cited 2007 Jul 4]. Available from: http://www.icn.ch/Acendio2007/ICNPcatalogues-042 107.html

12. Jansen K. ICNP ${ }^{\circledR}$ Catalogues. Presented in: ACENDIO Conference, 2007 apri 19-21. Amsterdam, Netherlands. [Cited 2007 Jul 4]. Available from: http://www.icn.ch/Acendio2007/ ICNPtutorial-Catalogues-041 907.html 